\title{
Poly(Vinyl Alcohol)-Infiltrated Carbon Nanotube Carpets
}

\author{
Konstantinos G. Dassios \\ Department of Materials Science and Engineering, University of Ioannina, Ioannina, Greece. \\ Email: kdassios@cc.uoi.gr
}

Received April 19 $9^{\text {th }}, 2012$; revised June $5^{\text {th }}, 2012$; accepted July $10^{\text {th }}, 2012$

\begin{abstract}
Carbon nanotube-polymer interaction is one of the key factors controlling the mechanical properties in composite and hybrid systems of such constituents. The current study reports a series of direct observations of substantial polymer sheathing phenomena on millimeters-high carpets of vertically aligned tubes infiltrated by Poly(vinyl alcohol) (PVA). SEM and TEM images of the composite's fracture surface revealed that sheathing was extensive and universal over the carpet's volume and did not influence the morphology, alignment or physical characteristics of the tubes. A significant increase in the tubes' diameters due to PVA coating was measured. Thermogravimetric analysis results were compatible with a crystallinity increase of the polymer phase due to the presence of CNTs, indicating the potential of CNTs in nucleating polymer crystallinity. Potential applications of the nanocomposite are discussed.
\end{abstract}

Keywords: Carbon Nanotubes; Polymer Nanocomposites; Thermogravimetric Analysis

\section{Introduction}

Owing to their exceptional physical properties such as strength, toughness and high aspect ratios, carbon nanotubes (CNTs) [1], have emerged as the most promising reinforcing medium for modern composite systems. In the nanocomposite material family, the efficiency and homogeneity of CNT dispersion within the matrix material are of equally high importance as understanding and controlling the interfacial interactions occurring between the nano-reinforcement and the continuous phase.

It is long now well established that CNTs have poor dispersibility in polymeric matrices unless their surface is previously chemically modified or elsewise functionalized $[2,3]$. Under this route, polymer matrix nanocomposites of mainly single CNT nanofibers have successively been manufactured [4-7], where the matrix material was mainly poly(vinyl alcohol) (PVA) [8-10], but also polypropylene and polyethylene [11-13]. Dalton et al. reported the production of super tough PVA/SWCNT fibers of a $60 \mathrm{wt} \%$ SWCNT content, processed with the aid of a surfactant [9]. However a straightforward methodology for achieving custom nanoreinforcement morphology and orientation within the continuous polymer medium is still being sought, mainly due to the difficulty associated with handling nanotubes and nanofibres. On the other hand, a detailed investigation of the polymer sheathing phenomenon in polymer/CNT nanocomposites has yet to be undertaken. Understanding the interaction between the two phases and the impact of sheathing on the physical properties of the interphase region is key to characterizing the mechanical properties of nanocomposites [14].

We present here direct evidence of substantial polymerMWCNT interaction in PVA/MWCNT nanocomposites produced by simple soaking, in PVA, of millimetres-high, as-grown carpets of vertically-aligned nanotubes that were previously wet by acetone alone, without having received any kind of chemical or physical modification. We found extensive polymer sheathing to have occurred throughout the product volume without alteration to the vertical alignment or morphology of the as-grown CNT carpet. Moreover, the tubes' physical characteristics remain unaffected by the interaction with the polymer that fills the open porosity of the carpet by coating annularly the tubes. The evidence presented herein includes:

1) SEM and TEM imaging of the carpets' volumes showing nanotubes coated annularly by PVA;

2) A 7 to 10-fold increase in the apparent nanotubes diameter due to sheathing measured at nanotube tips with protruding carbon cores;

3) TGA evidence supporting the argument that CNTs nucleate PVA crystallinity.

\section{Experimental}

\subsection{Synthesis of MWCNT Carpets}

The CVD methodology employed in this study makes use of a simultaneous catalyst/carbon feed concept to overcome the limitations of catalyst saturation-hence also final product height - encountered in the conventional catalyticCVD setups. Herein, the catalyst precursor, ferrocene and 
the carbon source, camphor, are introduced into the reactor in a common gas mixture so that, in the presence of fresh catalyst particles, carbon decomposition remains active for longer periods of time. The advantages of the simultaneous feed concept are manifold, as the technique allows for 1) Synthesis of unconventionally high CNT carpets; 2) Use of bare substrates, as opposed to conventional catalyst-coated ones and 3) Deposition on randomly-shaped substrates, as well as on non-silicon substrates. Another improvement to the conventional CVD setup is the burning of the gaseous product of the decomposition under open flame, before it is released to a HEPA-filter fume hood and then on to the environment. During this phase, the gas output is efficiently purged of phenanthrene, naphthalene and other polycyclic aromatic hydrocarbons (PAH). On the other hand, the CVD route employed for synthesizing the CNT carpets, based on commercial camphor, is economical, environmentally friendly and conforms with the 12 principles of green chemistry as issued by the United States Enviromental Protection Agency (US EPA) [15].

MWCNT carpets were synthesized inside a $1.2 \mathrm{~m}$ long quartz reactor, inner diameter $40 \mathrm{~mm}$, that was positioned horizontally in a three-zone split furnace (Figure 1). The carried gas, nitrogen, was introduced in the reactor at a flow rate of $430 \mathrm{ml} / \mathrm{m}$. The catalyst and carbon sources, ferrocene and camphor respectively, were mixed in solid state (powder form) in a 1:20 ratio and transferred to a glass flask that connected to the quartz reactor by means of a T-join. The reagents were evaporated with the help of a heater plate at $230^{\circ} \mathrm{C}$ and the vapors were carried by the nitrogen flux into the reactor. Crystalline silicon plates of 1-0-0 crystal orientation, and dimensions of $150 \mathrm{~mm} \times$ $38 \mathrm{~mm} \times 0.725 \mathrm{~mm}(1 \times \mathrm{w} \times \mathrm{t})$, were inserted centrally along the reactor's length and serve as substrates for the deposition of CNTs. The surface of the substrates was initially cleaned with acetone. Introduction of precursors into the reactor was allowed once the reactor temperature was stabilized at $850^{\circ} \mathrm{C}$. Chemical vapour deposition of carbon nanotubes endured for ca. $60 \mathrm{~min}$ and was terminated manually. Output gases were burnt under open flame before being released into a fume hood and then on to the environment. This step allows removal of harmful polycyclic aromatic hydrocarbons (PAH), such as naphthalene and phenanthrene, produced during the deposition. To the authors' knowledge, this is the first CNT growth reactor to implement a purification of the output gases which is a step closer to a cleaner environment. Upon removal of the substrates, thick deposits (ca. $2-2.5 \mathrm{~mm}$ in height for the stated experiment duration) of vertically-aligned MWCNTs were found on the Si wafers while the inner walls of the quartz reactor were also covered with thinner MWCNT layers. The MWCNT carpets were separated from the Si wafers using a razor blade. The maximum growth rate for a $2.3 \mathrm{~mm}$-high final product was calculated at 0.7 $\mu \mathrm{m} / \mathrm{sec}$.

\subsection{Fabrication of Nanocomposites and Samples}

A $10 \mathrm{wt} \%$ PVA solution was prepared by dissolution of commercial PVA in deionized water at $90^{\circ} \mathrm{C}$ and subsequent cooling to room temperature. Individual MWCNT carpets of approximate dimensions $40 \mathrm{~mm} \times 35 \mathrm{~mm}$ were soaked into pure acetone and then immersed into the PVA solution for 1 hour. Acetone acts as an intermediate medium to render the material hydrophilic - hence friendly to the water-based PVA solution - while it removes excess air trapped within the pores of the MWCNT carpet. The PVAsoaked samples were dried overnight in a vacuum oven.

The resulting PVA/MWCNT nanocomposite plates were cut into ca. $10 \mathrm{~mm} \times 10 \mathrm{~mm}$ pieces to be examined by Scanning Electron Microscopy, Transmission Electron Microscope and Thermo-Gravimetric Analysis. For TEM analysis, the PVA/MWCNT fibers were dispersed in DMF and sonicated for 5 minutes. Then a droplet of the solution was applied on a TEM copper/carbon grid. The grid was left to dry overnight at room temperature.

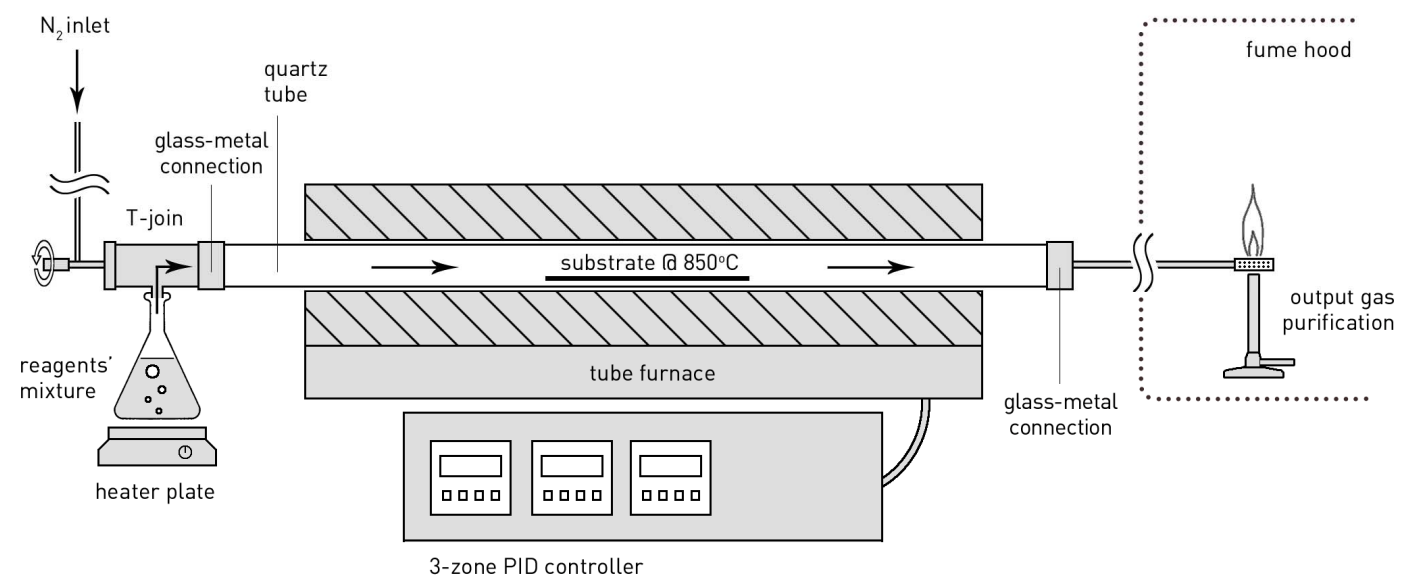

Figure 1. Thermal CVD setup for growth of MWCNT carpets of silicon substrates. 


\section{Results and Discussion}

Surprisingly, the only viable route for rendering the highlyhydrophobic as-grown tubes infiltratable by the aqueous PVA solution was wetting the carpets with acetone prior to transferring into the polymer. We believe that this unexpected finding is due to the absorption of acetone on the nanotubes' surface that enriches the nanotubes' surfaces with the oxygen atoms necessary in the formation of hydrogen bonds with the PVA radicals $[16,17]$. While polymer absorption was possible only on CNTs previously wet by acetone, it is important to recall that acetone itself is a poor solvent for PVA. Hence, acetone here is not a common mixing agent but only indirectly assists the sheathing mechanism, by providing a PVA-friendly oxygenrich coat onto the surfaces of individual carbon nanotubes.

A Zeiss SUPRA 35VP Scanning Electron Microscope with $30 \mathrm{kV}$ electron beam energy was used for the morphological characterization of the as-grown carpet and the nanocomposite. Carbon nanotubes in the as-grown carpet had diameters ranging between 30 and $50 \mathrm{~nm}$ and were aligned vertically with respect to the substrate (Figure 2). The typical morphology of the nanocomposite, as captured by SEM, is presented in Figure 3.

The first observation that emerged from the morphological examination of the composite carpets was that the surface of CNTs was the preferable location for absorption of PVA; polymer coagulates were not found in other locations. This means that the presence of the tubes did not allow the polymer to self-coagulate independently. Secondly, the polymer appeared to have deposited at annular layers around the tubes' surface, starting from the CNT surface; this observation was made possible at the free tips of PVA-coated tubes where concentric layers of polymer sheaths were visible around protruding cores of carbon nanotubes (Figure 3(b)). Similar observations were made possible in a Zeiss Libra-200 TEM, a typical high-resolution

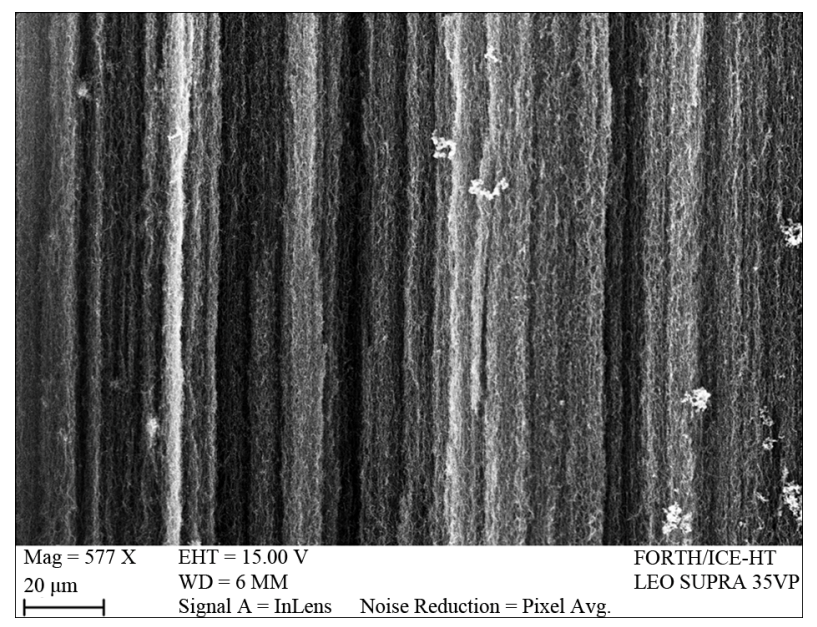

Figure 2. SEM micrograph of the as-grown carpet of vertically aligned CNTs.

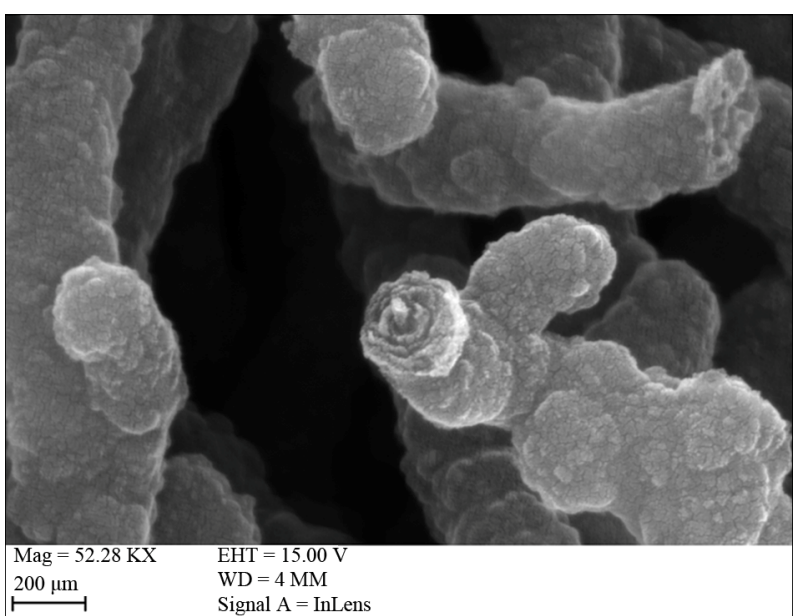

(a)

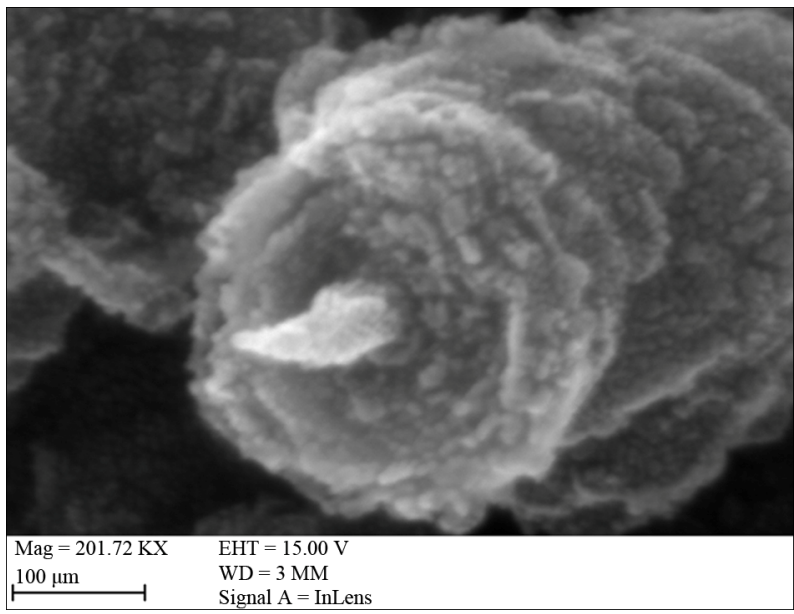

(b)

Figure 3. PVA-coated CNTs in the nanocomposite. (a) Morphology at low-magnification. Open circles demonstrate nanotube core tips exposed through the surrounding polymer; (b) Close-up of tips showing layers of PVA absorbed around the tubes.

image of the polymer sheathing phenomenon is demonstrated in Figure 4. CNT diameter in the composite carpet was measured at approximately $300 \mathrm{~nm}$, which is seven to ten timed greater than the as-grown tubes diameter. This "magnification" factor range has a significant engineering interest as it essentially controls the open porosity of the composite/hybrid material. This factor is controlled by the PVA concentration in the aqueous solution, which also controls the thickness of the polymer sheathe around the tubes.

A third observation was that the presence of the polymer did not promote coalescence of the nanotubes themselves nor changed the vertical alignment and morphology of the as-grown carpet. A similar behaviour has also been reported by Zhang et al. [6].

Thermogravimetric analysis (TGA) was employed to investigate the crystallinity and thermal response of the 
PVA/CNT nanocomposite as well as its constituents: 1) As-grown CNT carpet and 2) PVA in granule form (as received). As seen in Figure 5, owing to its fine structure, the as-grown carpet has the greatest thermal stability among the three materials. On the other hand, the PVA mass loss curve shows that the polymer has the lowest thermal stability, a property common to linear polymers of low molecular weight. The PVA curve exhibits two degradation stages: The first one at $300^{\circ} \mathrm{C}$ is related to the elimination of the amorphous parts of the polymer while the second one, at $400^{\circ} \mathrm{C}$, corresponds to the degradation of the higher thermal stability crystalline parts.

The nanocomposite's mass loss curve exhibited 5 steps of degradation: two significant drops at $520^{\circ} \mathrm{C}$ and $710^{\circ} \mathrm{C}$ and 3 intermediate steps at $580^{\circ} \mathrm{C}, 620^{\circ} \mathrm{C}$ and $650^{\circ} \mathrm{C}$ of less intensity. The first step, at $520^{\circ} \mathrm{C}$ is related to the decomposition of amorphous PVA parts, while the remaining multi-step behaviour can be attributed to the nucleated PVA crystallinity due to the presence of CNTs. Similar findings have been offered in the past for PVA/CNT mixtures of weight fractions as low as $0.1 \%$ [10] as well as

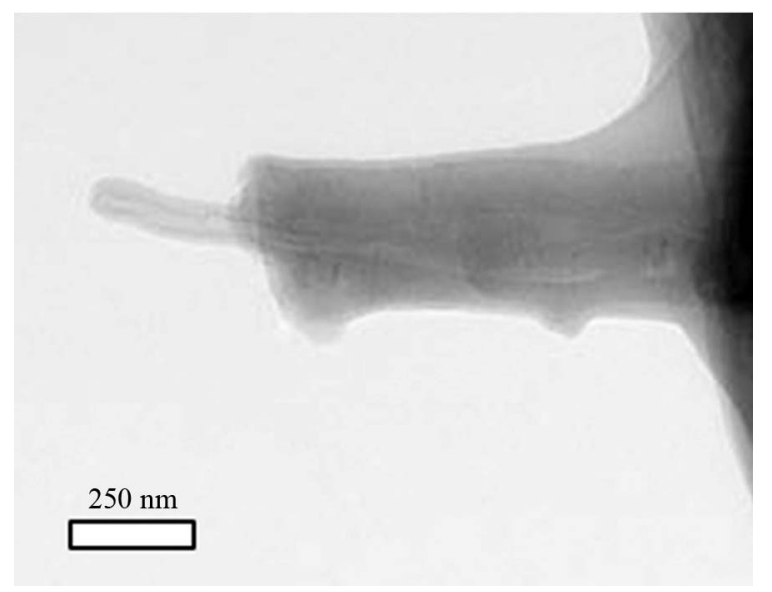

Figure 4. TEM image of PVA absorption around a carbon nanotube.

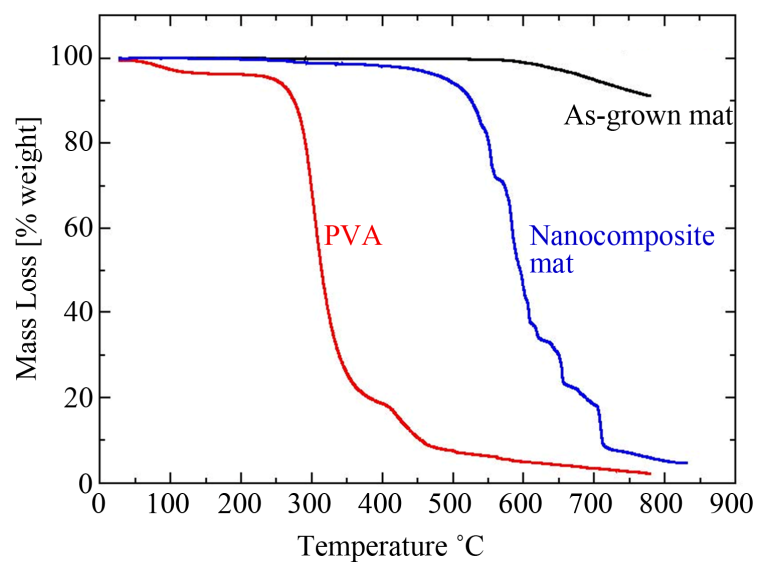

Figure 5. Mass change curves for the pristine CNT carpet, PVA and nanocomposite. for other polymers [18-20]. If crystallinity increases with decreasing nanotube diameter [4], the observed multi-step behaviour can be associated with crystallinity ranging from its highest value on small-diameter CNTs, to its lowest value at the outer layers of thicker tubes. Of course the diameter and crystallinity distributions are not discrete but continuous and therefore a one-to-one relation between mass drops in the TGA curve and the two affecting parameters should not be expected. This may explain the generally uneven form of the composite's TGA curve in (Figure 5). Nonetheless, it should be reminded that the PVA coating procedure did not involve thermal annealing, a procedure that could independently increase the degree of crystallinity of the polymer [21]. Hence the observed crystallinity nucleation is the sole result of the presence of CNTs.

\subsection{Potential Applications}

As discussed earlier, the polymer concentration in the drencher solution affects the thickness of the PVA sheath on the nanotubes; if this sheathing occurs annularly as shown herein, then this concentration eventually controls the open porosity of the composite. This aspect can be exploited in obtaining materials of custom porosity. For example, by using different polymer concentrations in the drencher solution, carpets of scalable pore size are achievable that could be used in filtering applications. Moreover, from the application point of view, the new hydrophilic character of the PVA-sheathed CNT carpet allows interaction with more materials. On the other end, the porosity of the carpet can be completely replaced by PVA to produce, for example, composite plates of z-reinforcements. This can be achieved either by using solutions of high PVA concentrations or by consecutive drenching on the carpet in lower concentration solutions. Such plates could be used as elements of sandwich panels.

From a mechanical performance point of view, the increase in PVA crystallinity on the CNT surface discussed earlier can provide significant improvement in mechanical properties. It has previously been shown that the addition of $0.5 \%$ DWCNT into PVA is responsible for a $1.3 \%$ increase in crystallinity and a three-fold increase in Young's modulus [7]. On the other hand, polymer-sheathed CNTs are promising materials for various sensing applications due to their large specific surface area and good electrical properties [22]. Aligned CNTs especially, once coated with conducting polymers can be used for producing glucose and complementary DNA sensors of high accuracy and selectivity.

PVA can also be utilized as an ideal protective/handling colloid for bulk CNTs in a manner similar to its use as sizing in common fibres. Lastly, PVA's aerobic and anaerobic biodegradability, a unique property among the polyvinyl-type synthetic polymers [23], could unambiguously 
be exploited in applications requiring recyclable, non-toxic and environmentally friendly nanocomposites.

\section{Conclusion}

PVA/MWCNT composite plates were produced by soaking millimetres-high CVD-grown carpets of verticallyaligned nanotubes into a $10 \mathrm{wt} \%$ PVA solution. The carpets were rendered hydrophilic by wetting with acetone alone, a process that endowed the tubes' surface with the oxygen necessary for the formation of hydrogen bonds with the PVA. Extensive polymer-CNT interaction in the form of polymer sheathing was directly observed in the nanoscale under SEM and TEM. It was found that the polymer did not interfere with the internal structure of the grown carpets and the nanotubes' physical characteristics remained unaltered by the sheathing mechanism. No PVA or CNT coagulates were found throughout the volume, an observation indicating that nanotubes interacted naturally with the polymer. The apparent diameters of annularly coated CNTs were found to be 7 - 10 larger than the diameters of as-grown tubes. Additional thermo-gravimetric examination of the nanocomposites showed evidence of variable PVA crystallinity as a pure result of the presence of CNTs, hence confirming the assertion that nanotubes can efficiently nucleate polymer crystallinity. The application perspectives of the produced nanocomposite include nanofilters of scalable pore size, full composite structural components for microdevices, sensing applications, biodegradable and environmentally-friendly components.

\section{REFERENCES}

[1] S. Iijima, "Helical Microtubules of Graphitic Carbon," Nature, Vol. 354, No. 6348, 1991, pp. 56-58. doi: $10.1038 / 354056 \mathrm{a} 0$

[2] K.-T. Lau and D. Hui, "Effectiveness of Using Carbon Nanotubes as Nano-Reinforcements for Advanced Composite Structures," Carbon, Vol. 40, No. 9, 2002, pp. 1605-1606. doi:10.1016/S0008-6223(02)00157-4

[3] V. Datsyuk, M. Kalyva, K. Papagelis, J. Parthenios, D. Tasis, A. Siokou, I. Kallitsis and C. Galiotis, "Chemical Oxidation of Multi Walled Carbon Nanotubes," Carbon, Vol. 46, No. 6, 2008, pp. 833-840. doi:10.1016/j.carbon.2008.02.012

[4] K. P. Ryan, et al., "Carbon Nanotubes for Reinforcement of Plastics. A Case Study with Poly(Vinyl Alcohol)," Composites Science and Technology, Vol. 67, No. 7-8, 2007, pp. 1640-1649. doi:10.1016/j.compscitech.2006.07.006

[5] X. Zhang, et al., "Poly(Vinyl Alcohol)/SWNT Composite Film," Nano Letters, Vol. 3, No. 9, 2003, pp. 1285-1288. doi:10.1021/n1034336t

[6] X. Zhang, et al., "Gel Spinning of PVA/SWNT Composite Fiber," Polymer, Vol. 45, No. 26, 2004, pp. 8801-8807. doi:10.1016/i.polymer.2004.10.048
[7] B. Vigolo, et al., "Improved Structure and Properties of Single-Wall Carbon Nanotube Spun Fibers," Applied Physics Letters, Vol. 81, 2002, pp. 1210-1212. doi:10.1063/1.1497706

[8] K. P. Ryan, et al., "Multiwalled Carbon Nanotube Nucleated Crystallization and Reinforcement in Poly(Vinyl Alcohol) Composites," Synthetic Metals, Vol. 156, No. 24, 2006, pp. 332-335. doi:10.1016/j.synthmet.2005.12.015

[9] P. Xue, et al., "Electrically Conductive Yarns Based on PVA/Carbon Nanotubes," Composites Structures, Vol. 78, No. 2, 2007, pp. 271-277.

[10] A. B. Dalton, et al., "Super-Tough Carbon-Nanotube Fibres," Nature, Vol. 423, 2003, p. 703. doi: $10.1038 / 423703 \mathrm{a}$

[11] O. Probst, E. M. Moore, D. E. Resasco and B. P. Grady, "Nucleation of Polyvinyl Alcohol Crystallization by Single-Walled Carbon Nanotubes," Polymer, Vol. 45, No. 13, 2004, pp. 4437-4443. doi:10.1016/j.polymer.2004.04.031

[12] M. Cadek, et al., "Morphological and Mechanical Properties of Carbon-Nanotube-Reinforced Semicrystalline and Amorphous Polymer Composites," Journal of Applied Physics Letters, Vol. 81, No. 27, 2003, p. 5123. doi:10.1063/1.1533118

[13] A. R. Bhattacharyya, et al., "Crystallization and Orientation Studies in Polypropylene/Single Wall Carbon Nanotube Composite," Polymer, Vol. 44, No. 8, 2003, pp. 2373-2377. doi:10.1016/S0032-3861(03)00073-9

[14] Z. Spitalsky, D.Tasis, K. Papagelis and C. Galiotis, "Carbon Nanotube-Polymer Composites: Chemistry, Processing, Mechanical and Electrical Properties," Progress in Polymer Science, Vol. 35, No. 3, 2010, pp. 357-401. doi:10.1016/j.progpolymsci.2009.09.003

[15] P. Anastas and J. C. Warner, "Green Chemistry: Theory and Practive," Oxford University Press, Oxford, 1998, p. 30.

[16] K. G. Dassios and C. Galiotis, "Polymer-Nanotube Interaction in MWCNT/Poly(Vinyl Alcohol) Composite Mats," Carbon, Vol. 50, No. 11, 2012, pp. 4291-4294. doi:10.1016/j.carbon.2012.04.042

[17] K. G. Dassios, S. Musso and C. Galiotis, "Compressive Behavior of MWCNT/Epoxy Composite Mats," Composites Science and Technology, Vol. 72, No. 9, 2012, pp. 1027-1033. doi:10.1016/j.compscitech.2012.03.016

[18] X. L. Xie, K. Aloys, X. P. Zhou and F. D. Zeng, "Ultrahigh Molecular Mass Polyethylene/Carbon Nanotube Composites - Crystallization and Melting Properties," Journal of Thermal Analyisis and Calorimetry, Vol. 74, No. 1, 2003, pp. 317-323. doi:10.1023/A:1026362727368

[19] J. Sandler, G. Broza, M. Nolte, K. Schulte, Y.-M. Lam and M. S. P. Shaffer, "Crystallization of Carbon Nanotube and Nanofiber Polypropylene Composites," Journal of Macromolecular Science, Part B: Physics, Vol. 42, No. 3-4, 2003, pp. 479-488.

[20] B. P. Grady, F. Pompeo, R. L. Shambaugh and D. E. Resasco, "Nucleation of Polypropylene Crystallization by Single-Walled Carbon Nanotubes," Journal of Physcal Chemistry B, Vol. 106, No. 23, 2002, pp. 5852-5858. 
doi:10.1021/jp014622y

[21] J. F. Kenney and G. W. Willcockson, "Structure-Property Relationships of Poly(Vinyl Alcohol). III. Relationships between Stereo-Regularity, Crystallinity, and Water Resistance in Poly(Vinyl Alcohol)," Journal of Polymer Science Part A-1: Polymer Chemistry, Vol. 4, No. 3, 1996, pp. 679-698. doi:10.1002/pol.1966.150040321

[22] D. Aussawasathien, P. He and L. Dai, "Polymer Nanofi- bers and Polymer Sheathed Carbon Nanotubes for Sensors," Polymeric Nanofibers, Chapter 18, 2006, pp. 246268.

[23] S. Matsumura, H. Kurita and H. Shimokobe, "Anaerobic Biodegradability of Polyvinyl Alcohol," Biotechnology Letters, Vol. 15, No. 7, 1993, pp. 749-754. doi:10.1007/BF01080150 